\title{
ESTUDIO SOBRE LA COMUNICACIÓN DIGITAL DE LAS ORGANIZACIONES SOCIALES EN ESPAÑA
}

\begin{abstract}
Ana Almansa-Martínez: Universidad de Málaga. España
\end{abstract} anaalmansa@uma.es

María Jesús Fernández-Torres: Universidad de Málaga. España mariajesusfernandez@uma.es

\section{RESUMEN}

Este artículo muestra el uso que las organizaciones sociales de España están haciendo de internet, de sus sitios webs y de blogs y redes sociales: si están utilizando la red para dar a conocer sus proyectos, si contienen secciones específicas para socios, si hacen campaña de captación de socios o recaudación de fondos on-line (fundraising), etc. Para realizar el estudio seleccionamos como muestra las organizaciones sociales con mayor número de socios - Cruz Roja Española, Médicos Sin Fronteras, Intermón Oxfam, Ayuda en Acción y UNICEF-. Asimismo, se analizan las organizaciones más pequeñas, para comprobar si las diferencias en el número de socios influyen en el uso de internet. La técnica de investigación que emplearemos es el análisis de contenido de las páginas webs, blogs y redes sociales de las ONG. Posteriormente, hacemos un análisis comparativo del uso que hacen de internet las distintas organizaciones. El interés de este estudio radica en que, en la actualidad, partidos políticos y muchos grupos de interés padecen el descrédito de la ciudadanía, como se ha puesto de manifiesto en España con el "Movimiento 15 M". En este contexto, organizaciones y movimientos sociales se convierten en un marcado exponente de la participación ciudadana.

PALABRAS CLAVE: Medios sociales - Comunicación 2.0 - ONG - Grupo de Interés Internet

\footnotetext{
${ }^{1}$ Autor correspondiente

Ana Almansa Martínez: Profesora de la Universidad de Málaga. Málaga. España

Correo: anaalmansa@uma.es
} 


\title{
DIGITAL COMMUNICATION STUDY ON SOCIAL ORGANIZATIONS IN SPAIN
}

\begin{abstract}
This article demonstrates the use of social organizations in Spain are making internet, its websites and blogs and social networks: they are using the network to publicize their projects, if they contain specific sections for members, if they make campaign membership recruitment and fundraising on-line. For the study sample selected as social organizations with more partners - Cruz Roja Española, Médicos Sin Fronteras, Intermón Oxfam, Ayuda en Acción y UNICEF-. We also analyze smaller organizations, to test whether differences in the number of partners influencing Internet use. The research technique employed is content analysis of web pages, blogs and social networks of NGOs. Later, we make a comparative analysis of Internet use are different organizations. The interest of this study is that, today, many political parties and interest groups have the discredit of the citizenry, as shown in Spain with the "Movimiento $15 \mathrm{M}$ ". In this context, organizations and social movements become a strong exponent of citizen participation.
\end{abstract}

KEY WORDS: Social Media - Communication 2.0 - NGO - Interest Group - Internet

\section{INTRODUCCIÓN}

En la última década, se ha generalizado en nuestro país, y en muchos otros, el uso de internet. Vivimos en la "sociedad red", en la que la comunicación deviene en modernidad y es casi imprescindible para cualquier tipo de organización (Castells, 2006).

En este tipo de sociedad, las organizaciones ya han descubierto la "ventana abierta al mundo" que puede suponer un sitio web y están presentes, mayoritariamente, en la red. Además, actualmente, en la red no se va a estar únicamente con un sitio web, sino que blogs y redes sociales se han convertido en grandes exponentes de la comunicación on-line de las organizaciones.

En el caso de las organizaciones sociales, internet también ha supuesto una gran oportunidad, ya que le permite un contacto casi permanente con sus principales públicos a unos costes reducidos. 
campaña de captación de socios o recaudación de fondos on-line (fundraising).

Concretamente, las preguntas de las que partimos son ¿utilizan las organizaciones sociales internet para captar socios y recaudar fondos?, ¿influye el número de socios en el uso que se hace de internet?

Los objetivos de este estudio son conocer el uso que hacen de su websites las organizaciones sociales de España; descubrir si internet está sirviendo a las organizaciones sociales para difundir información a sus socios y para captar nuevos socios; y comprobar si las organizaciones sociales hacen fundraising en su website.

La hipótesis de partida es que el número de socios no influye en la presencia de las organizaciones en internet, aunque sí en el uso que hacen de la red.

\section{METODOLOGÍA}

Seleccionamos como muestra las organizaciones sociales para el desarrollo con mayor y menor número de socios, según la Guía de ONG de Eroski Consumer de septiembre de 20102, una de las últimas publicaciones que se han hecho sobre el sector en España.

De este modo, se analizan las cinco organizaciones sociales con más socios en España, que son Cruz Roja Española (909.126 socios), Médicos Sin Fronteras (487.722), Intermón Oxfam (240.814), Ayuda en Acción (204.570) y UNICEF (217.000); y las organizaciones sociales con menos socios contempladas en el estudio de Eroski Consumer, que son Vols (195 socios), Solman (172), Conosud (121), Fundación Amigó (100) y AFS Intercultura (98).

Se analizan las organizaciones con más socios y con menos socios para poder comparar los dos grupos y comprobar si el número de socios (el tamaño de la organización) influye en el uso que hacen de internet, cómo y para qué lo utilizan.

La técnica de investigación que empleada es el análisis de contenido de las páginas webs de las organizaciones sociales, de los blogs y redes sociales a las que te invitan desde el sitio web.

Con esta investigación se pretende comprobar, en primer lugar, si las organizaciones sociales utilizan internet para dar a conocer sus ideas, la causa por la que existen. Del mismo modo, como las organizaciones deben cuidar su comunicación con sus socios, analizamos si ofrecen información específica para sus socios, secciones exclusivas para ellos, así como si animan a hacerse socio y facilitan este trámite. En la misma 
para una organización, si hacen recaudación de fondos (fundraising).

Por último, se revisa si las organizaciones invitan o no a visitar blogs corporativos desde el sitio web y qué tema son los que se suelen tratar en estos blogs. Asimismo, se comprueba en qué redes sociales está la organización y qué debates son los que se están planteando en redes. El análisis de contenido se ha llevado a cabo en junio de 2011.

\section{ANÁLISIS Y DISCUSIÓN}

\subsection{Las organizaciones sociales para el desarrollo}

Este estudio se centra en las ONG que trabajan en el ámbito del Desarrollo. Por ello, es conveniente acotar el concepto y definir las ONG como "asociaciones que manifiestan las inquietudes y pensamientos de los individuos de una sociedad, presentándose como expresión de la comunidad civil a través de agrupaciones que defienden un ideal, un pensamiento común a una serie de personas" (Fernández Torres, 2004, p.134). La autora recurre a la Carta de las Naciones Unidas (1950) para recordar que las ONG nacen como "organizaciones internacionales cuya constitución no sea consecuencia de un tratado internacional" (Fernández Torres, 2004, p. 134).

Por su parte, Marcuello Servós matiza que las organizaciones no gubernamentales para el desarrollo (ONGD) son:

Organizaciones de carácter social, independientes y autónomas, jurídicamente fundadas y que actúan sin finalidad de lucro. Su acción se orienta hacia la cooperación al desarrollo y hacia la búsqueda de acuerdos de ayudas entre gobiernos con el objetivo de provocar la solidaridad y promover el desarrollo en los pueblos y sociedades del Tercer Mundo. (Marcuello Servós, 2007, p. 17).

Las organizaciones no gubernamentales son grupos de interés se agrupan personas con una misma sensibilidad social, con un planteamiento común que les une -que devienen en grupos de presión al llevar a cabo ciertas estrategias para intentar conseguir sus fines, generalmente presionando sobre las instancias de poder (Castillo, 2010).

Cuando el grupo de interés pasa a la acción, se transforma en grupo de presión. Sin embargo, no todos los grupos de interés ejercerán presión, por lo que no a todos podemos considerarlos grupos de presión (Castillo, 2001, p. 25). 
Suelen aparecer como empresas especializadas en la presión política, pero sin depender intereses propios, sino aquellos por los que le han contratado (Castillo, 2001, p. 31-39).

\subsection{La comunicación de las ONG}

Las ONG, como acabamos de ver, surgen para reivindicar una causa o ideal. Por eso, para lograr su objetivo, necesitan sensibilizar sobre esa causa. Y para lograrlo, le resulta imprescindible la comunicación.

La comunicación va a permitir a las organizaciones la sensibilización sobre sus objetivos, así como desarrollar el conocimiento por parte del público del propósito y actividades de la organización (Cutlip y Center, 2001). Se pretende obtener la aceptación del interés común de todos los miembros de la organización. No sólo buscan la aceptación interna, sino también de la sociedad en general. Las organizaciones desarrollan canales de comunicación con sus públicos, inducen a los individuos a utilizar los servicios que la organización pone a su servicio crean y mantienen un clima favorable para la recaudación de fondos, crean materiales formativos, reclutan nuevos miembros, captar y forman al voluntariado, apoyan el desarrollo y mantenimiento de políticas públicas favorables a sus ideas, informan y motivan a sus públicos, intentan influir en la legislación y decisiones del gobierno para que se tomen los acuerdos que les sean más favorable, etc.

\subsection{Métodos de operación de las ONG}

En primer lugar están las manifestaciones masivas, como herramienta que tiene la ONG de exponer el apoyo popular a una causa determinada e intentar que haya un nuevo planteamiento al respecto (Wilcox, Autt, Agee y Cameron, 2001). En la misma línea están las concentraciones, sentadas o encierros.

Las ONG también pueden llevar a cabo iniciativas cuantitativas dirigidas a los representantes públicos, con el fin de ofrecer la imagen de que cuentan con un amplio sustento social. Este tipo de actuaciones pretenden implicar a los ciudadanos y que sean éstos los que envíen de forma individual sus mensajes a las personas que puedan decidir sobre las peticiones del grupo.

Otra forma de actuar es la reconciliación o negociación, manteniendo contacto con el gobierno, empresas vinculadas al interés o causa de la ONG.

Cuando el acuerdo no es posible, las organizaciones pueden recurrir al boicot. Las 
Las organizaciones pueden interponen demandas. Recurren al litigio, interponen demandas con la intención de que la jurisprudencia les sea favorable y se convierta en norma aquello que reivindican.

Las ONG también recurren a la espectacularidad. Para llamar la atención, muchas organizaciones vienen recurriendo a la espectacularidad, al protagonizar actos de protesta muy llamativos. También las organizaciones intentan conseguir sus objetivos desde dentro de las empresas o instituciones es decir, formando parte de ellas, de su accionariado, y, desde dentro, introducir esos cambios que vienen reivindicando (métodos endógenos). Evidentemente, sólo las organizaciones con más recursos pueden llevar a cabo este tipo de actuaciones endógenas.

Lo que sí hace cualquier ONG es cuidar la relación con los medios de comunicación. Los medios de comunicación van a permitir a las organizaciones cumplir una serie de funciones (Castillo, 2001, p. 244):

a) Función difusora. Difundir entre los ciudadanos sus propuestas y demandas.

b) Función movilizadora entre sus simpatizantes y la ciudadanía en general.

c) Función de cohesión psíquica, con la intención de aglutinar, aunar.

d) Función de apoyo social, dar la imagen a través de los medios de que el grupo cuenta con apoyo.

e) Función educativa, presentar instrumentos instructivos sobre las problemáticas.

f) Función sensibilizadora, actuar como elementos sensibilizadores un tema en cuestión.

g) Función restrictiva, limitar el acceso mediático a intereses divergentes.

h) Función de relaciones públicas, mejorar la imagen del grupo.

i) Función monopolizadora, presentarse y convertirse como la organización especialista en determinada cuestión.

j) Función legitimadora, al aparecer en los medios de forma frecuente las organizaciones no gubernamentales se van legitimando.

No todos los grupos de presión consiguen desarrollar todas las funciones antes mencionadas. Por ejemplo, la función restrictiva sólo es posible en escasas ocasiones.

Por otro lado, las ONG suelen editar folletos, publicaciones periódicas y boletines que explican los objetivos de la organización y las actividades que desarrollan.

Asimismo, las organizaciones sociales suelen organizar eventos como herramienta 
Los recaudadores de fondos o fundraisers saben muy bien que los ciudadanos donan dinero por el simple deseo de compartir, por ego (por la forma cómo lo agradecerá la organización, con la puesta de una placa, hacer pública la lista de donantes, etc.) o lo que se conoce como la presión de los pares, es decir, lo difícil que resulta negarse a la petición de un amigo.

Los métodos tradicionales de recaudación de fondos son los telemaratones, la venta de objetos, la captación de donativos, la organización de campañas de recaudación con múltiples eventos, rifas, cenas y patrocinios de acontecimientos, pedir donativos por teléfono, ofrecer una línea 900 de llamada gratuita para que los donantes lo hagan sin coste alguno, iniciativas comerciales (como dar licencia para que un producto se comercialice con el nombre de la organización -Unicef lo hace-, o gestionar negocios propios -ropas de segunda mano-, etc.) o el correo directo (cada vez más por correo electrónico). Por otro lado, las ONG también están haciendo fundraising en su página web, aprovechando las oportunidades que les ofrece internet. Asimismo, la mayoría de las organizaciones reciben cuotas de sus socios, que también les ayuda a financiarse (y también se captan socios por medio de su website).

\subsection{Aportaciones de las nuevas tecnologías}

Las nuevas tecnologías han aportado rapidez y comodidad, entre otras ventajas, al quehacer cotidiano de la comunicación. Los sitios web han supuesto grandes ventajas y oportunidades para quienes gestionan la comunicación. Principalmente son tres (Seitel, 2002). La primera es que internet permite ofrecer a públicos amplios y dispersos extensa información sobre las organizaciones y sus proyectos. El segundo aspecto que destaca Seitel es que se puede trabajar en tiempo real, la inmediatez y la posibilidad de respuesta inmediata. Y el tercer aspecto que destaca Seitel es el de la segmentación, posible en internet y que supone una mayor efectividad en comunicación. Adecuar el mensaje al público al que se dirige ayuda a que la comunicación sea más efectiva.

Las aportaciones de las nuevas tecnologías y de internet a la comunicación de las organizaciones se pueden resumir de la siguiente manera: rapidez a la hora de localizar y difundir información; permite incorporar motores de búsqueda, haciendo más cómoda y rápida la consulta; posibilidad de actualizaciones constantes; interactividad con los públicos; gran capacidad de almacenamiento; posibilidad de incluir recursos multimedia, que suponen un valor añadido (sin duda, puede resultar más atractivo y fácilmente comprensible que, por ejemplo, el uso exclusivo del texto); no hay limitación temporal de consulta y se puede acceder desde cualquier lugar; y 
Por otro lado, la Really Simple Syndication (RSS) es, sin duda, una de más exitosas incorporaciones a las páginas webs. (González-Herrero \& Ruiz de Valbuena, 2006, p.267-275). Permite suscripciones a la página web por parte de los ciudadanos y que éstos reciban vía electrónica las actualizaciones que se puedan hacer en la web.

Algo parecido sucede con las salas de prensa virtuales o press room, las secciones de las web corporativas que están dirigidas específicamente a los medios de comunicación, uno de los públicos prioritarios para cualquier organización. Ahora, la relación con los medios podrá ser más fluida y la organización podrá facilitar de forma cómoda recursos (video, audio, etc.) impensables con medios tradicionales.

Y es que, en la actualidad, ninguna organización puede obviar el poder de convocatoria y la capacidad de movilización que tienen las nuevas tecnologías (e internet en particular). Las grandes concentraciones se hacen utilizando la red. Muestra de ellos es el Movimiento $15 \mathrm{M}$.

Sin embargo, podemos plantearnos si, las organizaciones están aprovechando todo el potencial que supone internet, así como si el uso que se está haciendo las distintas ONG es semejante.

\subsection{Las ONGD españolas en internet}

Todas las organizaciones analizadas, tanto las grandes como las pequeñas, disponen de página web y su dirección coincide con el nombre de la organización. Es decir, las organizaciones sociales están utilizando la red como la ventana abierta al mundo que es, independientemente del tamaño que tenga la organización.

Y es que, con la evolución de las últimas tecnologías, disponer de un sitio web ya no supone un gasto excesivo e incluso se dispone de plataformas que permiten la autogestión de webs y blogs de forma gratuita y sin necesidad de disponer de conocimientos informáticos elevados.

Del mismo modo, todas las organizaciones están aprovechando esta "ventana" para dar a conocer sus proyectos, sus ideas, su lucha a la ciudadanía en general. Existen diferencias en la forma como dan a conocer sus actuaciones, como comprobaremos más adelante, pero todas utilizan la web para difundir sus principales ideas y proyectos, así como para darse a conocer como organización.

\subsection{Buscando la colaboración ciudadana}


De hecho, curiosamente es la más pequeña organización del estudio (AFS Intercultura) la única ONG del grupo de las pequeñas que dispone de sección específica para los socios. Por su parte, todas las grandes (excepto Intermón Oxfam) disponen de sección específica para los socios, con los contenidos abiertos para todos los públicos, salvo en el caso de Cruz Roja y de Médicos Sin Fronteras que disponen de una intranet para socios a la que se accede mediante clave.

Todas las grandes organizaciones invitan a los visitantes de sus web a hacerse socios, se indica cómo hacerlo y, en algunos casos, es un trámite que se puede hacer on-line en la misma web (así lo hace, por ejemplo, Médicos Sin Fronteras). En el caso de las pequeñas organizaciones, la mayoría también se ocupa de la captación de socios. En realidad, todas las organizaciones lo hacen, menos Solman.

Igualmente, todas las organizaciones (incluidas grandes y pequeñas), menos una (Conosud), intentan reclutar voluntarios sus páginas web. Animan a ser voluntario/a y se indica qué hacer para empezar a colaborar. La única organización que no recluta voluntariado desde su web, Conosud, es una de las más pequeñas.

La mayoría de las organizaciones estudiadas pretenden recaudar fondos a través de su página web. Conocedoras de que necesitan hacer fundraising para su propia supervivencia, van a aprovechar la oportunidad que les da la red. Sin embargo, en este aspecto también existen diferencias entre grandes y pequeñas. Todas las grandes organizaciones recaudan fondos para su causa en general o para proyectos en concreto (algunas hacen recaudación para la organización en general y para proyectos concretos). Sin embargo, el caso de las pequeñas es distinto, ya que encontramos dos ONG -Conosud y AFS Intercultura- que no hacen ningún tipo de recaudación en sus web.

Evidentemente, para recaudar fondos, las organizaciones van a dar todo tipo de facilidades a los ciudadanos, siendo bastante habitual que el proceso se puede desarrollar íntegramente en la red. Un buen ejemplo de ello es Unicef, que permite seleccionar la campaña concreta para la que se dona.

Fundraising, voluntariado, captación de socios... lo que todas las organizaciones hacen en buscar la participación, la colaboración de los ciudadanos.

Algunas organizaciones, como es el caso de Ayuda en Acción, concentra sus esfuerzos en el apadrinamiento, convirtiéndose en la forma de colaboración ciudadana que más demanda desde su web. 


\subsection{Cómo aprovechan el potencial de internet}

Algunos potenciales de internet, como es el caso de la posibilidad de incorporación de recursos multimedia, no existen demasiadas diferencias entre grandes y pequeñas organizaciones. Sin embargo, sí que las hay en la actualización de la web.

Todas las organizaciones grandes, excepto Unicef, cuentan en su página web con recursos multimedia: videos, infografía y audio (los cortes de audio no son tan frecuentes, aunque organizaciones como Ayuda en Acción los incorpora). También todas las organizaciones pequeñas, excepto Conosud, tienen recursos multimedia en sus web. Es decir, tanto las grandes como las pequeñas organizaciones sociales, el $80 \%$ de ellas, cuenta con recursos multimedia. La diferencia radica en el $20 \%$ que no es multimedia, ya que Unicef presenta una página visualmente muy atractiva, con imágenes fijas, mientras que Conosud tiene una página en la que prácticamente lo único que hay es texto.

Tampoco hay demasiadas diferencias entre grandes y pequeñas organizaciones en cuanto a la interactividad. La mayoría de las organizaciones no disponen ni de chat, ni de foros, ni de encuestas ni de otros elementos interactivos. Parece que las organizaciones están apostando por la interactividad por medio de sus blogs y redes sociales, no utilizando tanto los medios más tradicionales de interactividad antes comentados.

Las excepciones que confirman la regla son Cruz Roja (entre las grandes) y Solman (entre las pequeñas). Cruz Roja, a pesar de tener presencia en redes sociales, no renuncia a los chat y foros en su web. En el caso de Solman, también encontramos foros en los que poder debatir sobre diferentes temáticas vinculadas a la organización. En ninguna de las organizaciones estudiadas se ha encontrado ninguna encuesta.

Por otro lado, son las organizaciones pequeñas las que ofrecen link con páginas relacionadas o enlaces de interés con mayor frecuencia: mientras que casi todas las organizaciones pequeñas lo hacen, prácticamente ninguna grande cuenta con enlaces a sitios externos.

Lo que sí que hacen todas las organizaciones, grandes y pequeñas, es ofrecer formas de contactar con ellas. La práctica totalidad de las ONG estudiadas ofrece correos electrónicos, teléfonos y direcciones postales como forma de contacto.

Todas las grandes organizaciones analizadas cuentan con buscador y mapa web como instrumentos de búsqueda en la web. Sin embargo, este tipo de ayudas escasean 
Asimismo, como ya hemos adelantado, existen diferencias en la actualización de la página web. Las grandes ONG actualizaron, en el periodo estudiado, su web al día y, en el peor de los casos, una vez a la semana. La actualización es mucho menor en el caso de las organizaciones pequeñas, que no renuevan contenidos en más de una semana. En el caso de las organizaciones pequeñas hay una excepción: AFS Intercultura, que hizo actualizaciones diarias.

Por otro lado, destaca la presencia de Sala de Prensa (Press Room) en la web de dos grandes organizaciones: Intermón Oxfam y Unicef. Esta sección específica para los medios de comunicación únicamente está presente en estas dos ONG.

Tampoco es habitual que las web de las ONG cuenten con RSS (Really Simple Syndication, que permite suscripciones a la página web por parte de los ciudadanos y que éstos reciban vía electrónica las actualizaciones que se hacen en la web). Únicamente tres ONG permite este servicio: Médicos Sin Fronteras, Intermón Oxfam y Unicef.

\subsection{Blog y redes sociales}

Mientras que la presencia en redes sociales está bastante generalizada en todas las organizaciones (aunque cabe hacer algunas matizaciones) el uso de blog con fines corporativos es bastante desigual. La mayoría de las grandes organizaciones cuenta con blog, a los que se puede acceder desde la web, aunque únicamente Médicos Sin Fronteras enlaza blog desde su home o página principal. Sin embargo, en ninguna pequeña organización encontramos mención alguna a blog corporativos.

Los blog analizados (todos vinculados a grandes ONG) suelen ser de dirigentes de la organización y, en algún caso, de socios o personas relacionadas con la ONG. Los temas que se abordan están siempre centrados en la actividad de la ONG, sus proyectos o su oferta formativa.

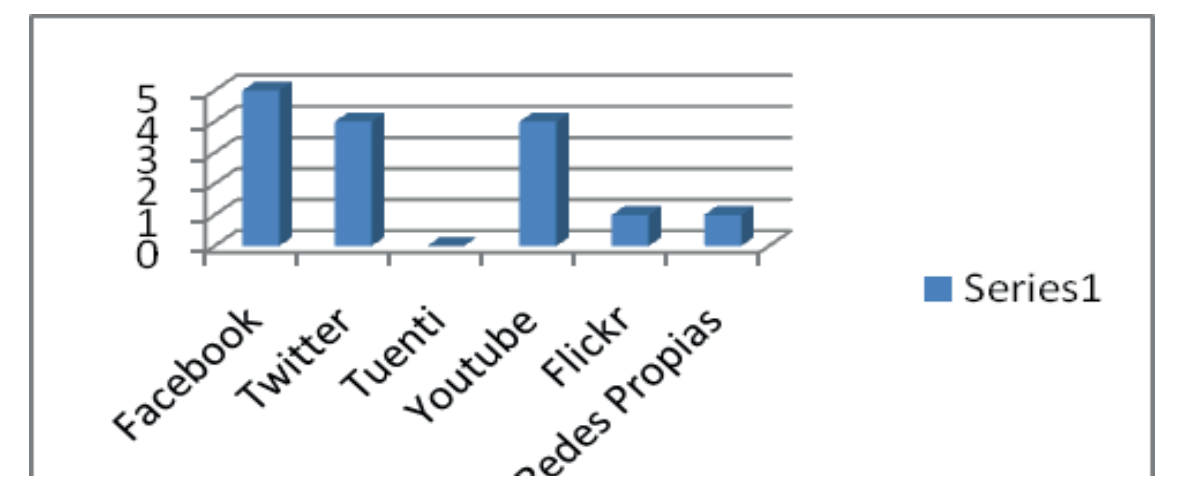


Parece que las organizaciones sociales prefieren las redes sociales para relacionarse con sus públicos. Prueba de ello es que todas las ONG estudiadas, excepto Fundación Amigó, están en redes sociales. Generalmente, desde la web se va a invitar al internauta a visitar el perfil de la organización en redes sociales, menos en los casos de Ayuda en Acción y de Conosud, que están en Facebook, pero no se indica en sus respectivas web.

Como se puede apreciar en los gráficos, existen diferencias considerables en la presencia en redes sociales. Facebook es la red social en la que están casi todas las organizaciones (grandes y pequeñas). En realidad, todas las ONG están en Facebook, menos Fundación Amigó. En segundo lugar, Twitter y Youtube parecen ser las preferidas y casi la totalidad de las grandes organizaciones están en estas redes (4 de 5), mientras que en el caso de las pequeñas organizaciones la presencia es de menos de la mitad (2 de 5). Tuenti es la red social que sale peor parada, ya que ninguna ONG de las estudiadas está en esta red.

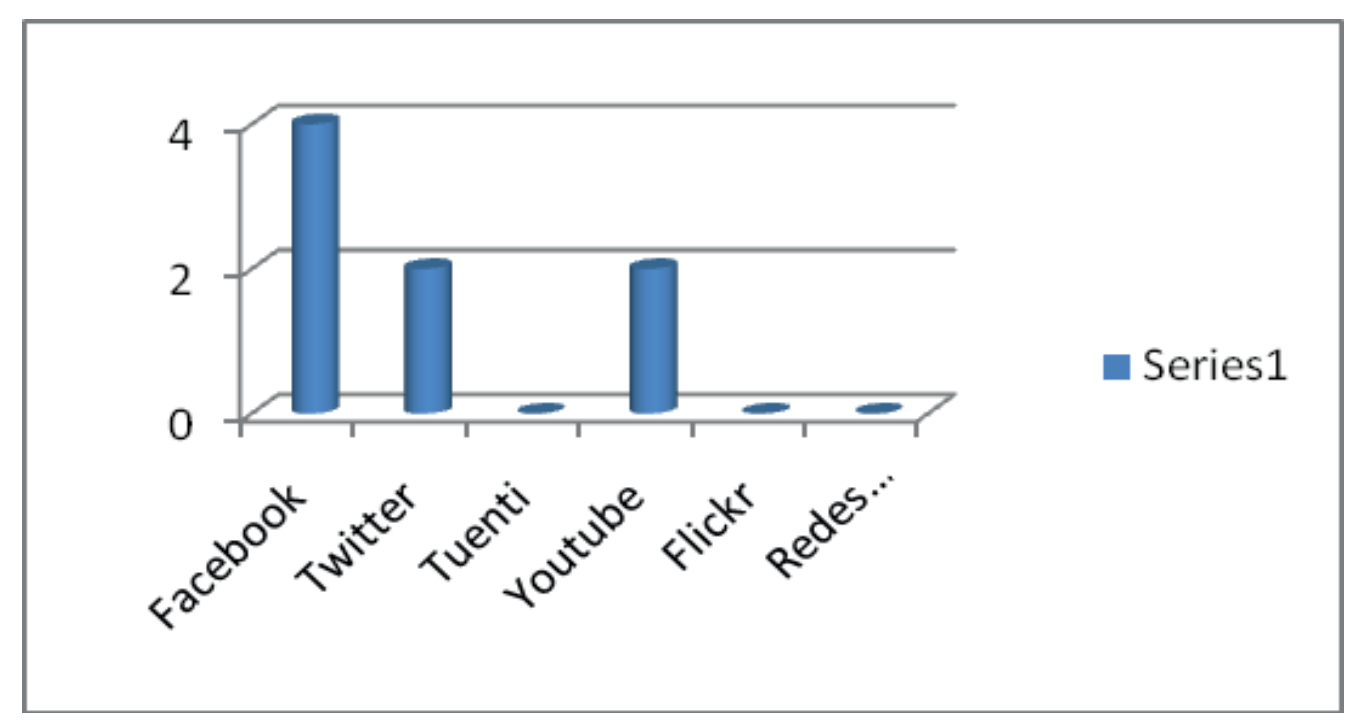

Figura 2. Presencia en redes sociales de las pequeñas organizaciones Fuente: Elaboración propia

Por último, hay que comentar la existencia de una red social propia en el caso de Cruz Roja: Mundo Cruz Roja. En las redes sociales, tanto las grandes como las pequeñas organizaciones, difunden las actividades que están llevando a cabo, presentan sus campañas, así como los proyectos en los que están inmersos. 


\section{CONCLUSIONES}

Las ONG españolas han descubierto las ventajas que para ellas tiene estar en internet. Por ello, independientemente del tamaño de la organización, disponen de sitio web en el que poder comunicarse con sus públicos prioritarios.

Del mismo modo, todas las organizaciones están aprovechando internet para dar a conocer sus proyectos, sus ideas, su lucha, a la ciudadanía en general; casi siempre, ofreciendo recursos multimedia. Y prácticamente todas las organizaciones, grandes y pequeñas, intentan captar socios, voluntarios y buscan cualquier tipo de colaboración a través de su página web, facilitando el contacto con la ONG.

Sin embargo, sí que existen diferencias evidentes entre grandes y pequeñas organizaciones a la hora de utilizar las opciones que les ofrece internet. De este modo, las diferencias comienzan con la forma cómo se relacionan con los socios: son las grandes las que, casi en exclusiva, van a contar con una sección específica para que el socio disponga de la información que precise.

También el fundraising, básico para las ONG, muestra diferencias entre grandes y pequeñas organizaciones. Mientras todas las grandes organizaciones recaudan fondos para su causa en general y/o para proyectos en concreto, casi la mitad de las pequeñas organizaciones no hace ningún tipo de recaudación en sus web.

Otro de los aspectos en los que difieren grandes y pequeñas ONGD es en las actualizaciones. Las grandes ONG actualizaron, en el periodo estudiado, su web al día y, en el peor de los casos, una vez a la semana. La actualización es mucho menor en el caso de las organizaciones pequeñas, que no renuevan contenidos en más de una semana. Tampoco las pequeñas organizaciones facilitan la navegación mediante buscadores y mapas web que, sin embargo, sí que están habitualmente presentes en las grandes ONG. Y sólo ONG grandes cuentan con RSS y Salas de Prensa Virtuales.

Las diferencias también son evidentes en el uso de blog corporativos, ya que la mayoría de las grandes ONGD dispone de este tipo de publicaciones corporativas, mientras que en las pequeñas no se encuentra ninguna. Sin embargo, la presencia en redes sociales está más generalizada y todas las ONG estudiadas, menos una, están en redes sociales. Facebook, Twitter y Youtube son las redes en las que están más presentes las ONGD.

Por todo lo anteriormente expuesto, podemos confirmar nuestra hipótesis de partida, que señalaba que el número de socios no influye en la presencia de las 
Como futura investigación, para completar el estudio que ahora se presenta, sería interesante llevar a cabo un análisis similar con otros actores políticos, para comprobar si en España hay organizaciones políticas, como pueden ser los partidos políticos, que hacen un mayor y mejor uso de internet o, por el contario, el panorama es similar entre los distintos actores políticos españoles.

\section{REFERENCIAS}

Afs Intercultura. (2011) Recuperado el 12 al 25 de junio de 2011, de http:/ / www.afsintercultura.org/esp_es/home.

Asociación Española de Fundraising. (2010). Principios básicos para la organización de eventos de captación de fondos. Madrid: Asociación Española de Fundraising.

Ayuda en acción. (2011) Recuperado el 12 al 25 de junio de 2011, de http://www.ayudaenaccion.org/index.asp.

Cabero Almenara, J. \& Córdoba Pérez, M. (2009). Educación Inclusiva. La educación digital. Revista de Educación Inclusiva, 2(1). Recuperado el 20 de mayo de 2011, de http://www.ujaen.es/revista/rei/linked/documentos/documentos/2-4.pdf.

Carrascosa, J. L. (1992). Comunicacción. Una comunicación eficaz para el éxito en los negocios. Madrid: Ciencias de la Dirección.

Castells, M. (2006). La sociedad red. Madrid: Alianza Editorial.

Castillo Esparcia, A. (2001). Los grupos de presión ante la sociedad de la comunicación. Málaga: Universidad de Málaga.

Castillo Esparcia, A. (2005). Relaciones públicas y tecnología de la comunicación. Análisis de las salas de prensa virtuales. Organicom, 2(3).

Castillo Esparcia, A. (2011). Lobby y comunicación. Sevilla: Comunicación Social.

Conosud. (2011) Recuperado el 12 al 25 de junio de 2011, de http://www.conosud.org/.

Cruz Roja Española. (2011) Recuperado el 12 al 25 de junio de 2011, de httn / / wow cri1zrnia es / nnrtada / 
Fajula Payet, A. \& Roca Correa, D. (2001). Las nuevas tecnologías de la comunicación (aplicación y repercusiones en el entorno empresarial. En AA.VV. Dirección de Comunicación empresarial e institucional. Barcelona: Gestión 2000.

Fernández Torres, M. J. (2004). Las relaciones públicas como gestión de la comunicación en los movimientos sociales. Análisis de la estrategia de las ONG en España. Málaga: Universidad de Málaga.

Fundación amigó. Recuperado el 12 al 25 de junio de 2011, de http://www.fundacionamigo.org/.

Gonzalez-Herrero, A. \& Ruiz de Valbuena, M. (2006). Trends in online media relations: web-based corporate press romos in leading international companies. Public Relations Review, 32. Recuperado el 15 de mayo de 2007, de http://www.sciencedirect.com/science/article/pii/S0363811106000580.

Grunig, J. \& Hunt, T. (2000). Dirección de Relaciones Públicas. Barcelona: Gestión 2000.

Intermón oxfam. (2011). Recuperado el 12 al 25 de junio de 2011, de http://www.intermonoxfam.org/es/page.asp?id=1.

Marcella, R.; Baxter, G. \& Moore, N. (2002). Theoretical and methodological approaches to the study of information need in the context of the impact of new information and communications technologies on the communication of parliamentary information. Journal of Documetation Emerald, 58(2).

Marcuello Servós, C. (2007).Capital social y organizaciones no lucrativas en España. El caso de las ONGD. Bilbao: Fundación BBVA.

Médicos sin fronteras. (2011). Recuperado el 12 al 25 de junio de 2011, de http://www.msf.es/.

Middleberg, D. (2001). Relaciones públicas en un mundo interconectado. Pamplona: Deusto.

Nafría, I. (2008). El usuario, el nuevo rey de internet. Barcelona: Gestión 2000.

Norris, P. (2002). Political parties, Social Capital and Civic Society, Conclusion, en Democratic Phoenix. Reinventing Political Activism. Madrid: Taurus. 
Mendiluce, J. M. (1998). La gestión de las organizaciones no lucrativas. Bilbao: Ed. Deusto.

SOLMAN. (2011). Recuperado el 12 al 25 de junio de 2011, de http:/ / www.solmanongd.org/.

UNICEF. (2011). Recuperado el 12 al 25 de junio de 2011, de http:/ / www.unicef.es/.

VOLS. (2011). Recuperado el 12 al 25 de junio de 2011, de http://www.ongvols.org/es/.

Wilcox, D.; Autt, P.; Agee, W. \& Cameron, G. (2001). Relaciones Públicas. Estrategias y Técnicas. Madrid: Addison Wesley.

\section{Ana Almansa Martínez}

Profesora en el Departamento Comunicación Audiovisual y Publicidad, en la facultad de Ciencias de la Comunicación de la Universidad de Málaga. Asimismo, es docente en postgrados de la Universidad de Almería, de la Universidad Mayor de Chile y de la Universidad de Málaga. Directora académica y docente del Curso de Experto sobre Comunicación, Protocolo y Organización de Actos, de la Universidad de Málaga; y directora académica de los Cursos de Especialización Universitaria Comunicación para la Igualdad. Su línea de investigación es la comunicación institucional, empresarial y organizacional y la incidencia que en estos ámbitos están teniendo las nuevas tecnologías.

\section{María Jesús Fernández Torres}

Doctora en Ciencias de la Comunicación por la Universidad de Málaga en 2004, con una tesis que versó sobre Movimientos Sociales y estrategias de comunicación en las ONG. Fue, en su momento, la Doctora más joven de España. Técnico Auxiliar en Protocolo. Especializada en Comunicación Interna y Externa. Funcionaria de carrera y profesora de la Universidad de Málaga. 